ISBN 978-981-14-1684-2

Proceedings of 2019 the 9th International Workshop on Computer Science and Engineering

(WCSE 2019 SUMMER)

Hong Kong, 15-17 June, 2019, pp. 370-377

doi: $10.18178 /$ wcse. 2019.06 .056

\title{
Research on Plant Growth Environment Control System Based on BP Neural Network
}

\author{
Meng $\mathrm{Li}^{1+}$, Liguo Tian ${ }^{1}$, Chuang Liu ${ }^{1}$, Hang Ding ${ }^{1}$ \\ ${ }^{1}$ Tianjin Key Laboratory of Information Sensing \& Intelligent Control, Tianjin University \\ of Technology and Education, Tianjin, 300222, China
}

\begin{abstract}
As an advanced stage of modern facility agriculture development, plant factory is an efficient agricultural production system that realizes optimal plant growth by regulating plant growth environment. It mainly complies the automatic control of environmental factors such as temperature, humidity, illuminance and $\mathrm{CO}_{2}$ concentration during plant growth. With the application of neural network in nonlinear and strong coupling control systems, this paper establishes a plant growth environment control system based on LabVIEW. Depend on this platform, we study and establish an environmental state model based on BP neural network, and achieve precise plant growth environment control. The experimental result proves that the system runs stably and reliably and meets the design requirements.
\end{abstract}

Keywords: BP neural network, plant growth, control system, LabVIEW

\section{Introduction}

With the continuous development of modern agricultural technology, various control and regulate strategies of plant growth environment create the optimal environment condition for plant growth, which overcome the influence of temperature, irrigation, climate, season and other factors. Moreover, plant yield and economic benefits are improved, meanwhile, energy consumption and waste are reduced under the precondition that the ecological benefits are ensured. Plant factory is the advanced stage of modern facility agriculture development, how to achieve precise control and regulation of plant growth environment is the key research work that need to be solved.

The plant growth environment control system is a highly nonlinear, strongly coupled, multi-input and output system with dynamic characteristics, largely influenced by the external climate (wind speed, outdoor temperature and humidity) and many other practical constraints (executing agencies) interference. In this paper, through the study and research of the controllable environment optimization control method, a small plant growth environment simulation system-plant growth cabinet is established, and the plant growth environment control system is established, and the control method is mainly studied. The BP neural network technology is used to treat the plants. Self-learning and self-adaptation of the environmental parameters of the growth process, establishing a plant growth environment state model based on BP neural network, and obtaining the initial state of the plant growth environment, adjusting the actuator according to the environmental factors and the standard values, thereby maintaining the plant growth environment stability.

\section{Design of The Plant Growth Environment Control System}

\subsection{Overall Structure of System}

Plant growth depends on the external environment, and it needs external environment conditions such as temperature, humidity, illuminance and $\mathrm{CO}_{2}$ concentration necessary for growth. The experimental

\footnotetext{
+ Corresponding author. Tel.: + 15022270753

E-mail address: 1mtute2013@163.com.
} 
equipment built in this paper, the plant growth cabinet, has different sources of heterogeneous sensors arranged in the cabinet.

At the location, the information of each environmental factor in the cabinet is transmitted to the computer through the sensor, and the computer processes and analyzes the data, determines the state of the plant growth environment, and makes an instant judgment compared with the set standard value, thereby controlling the actuator, thereby Environmental factors such as temperature, humidity, illuminance and $\mathrm{CO} 2$ concentration in the plant growth environment are adjusted to suit the environmental factors required for plant growth. The structural diagram of control system can be shown as figure 1 .

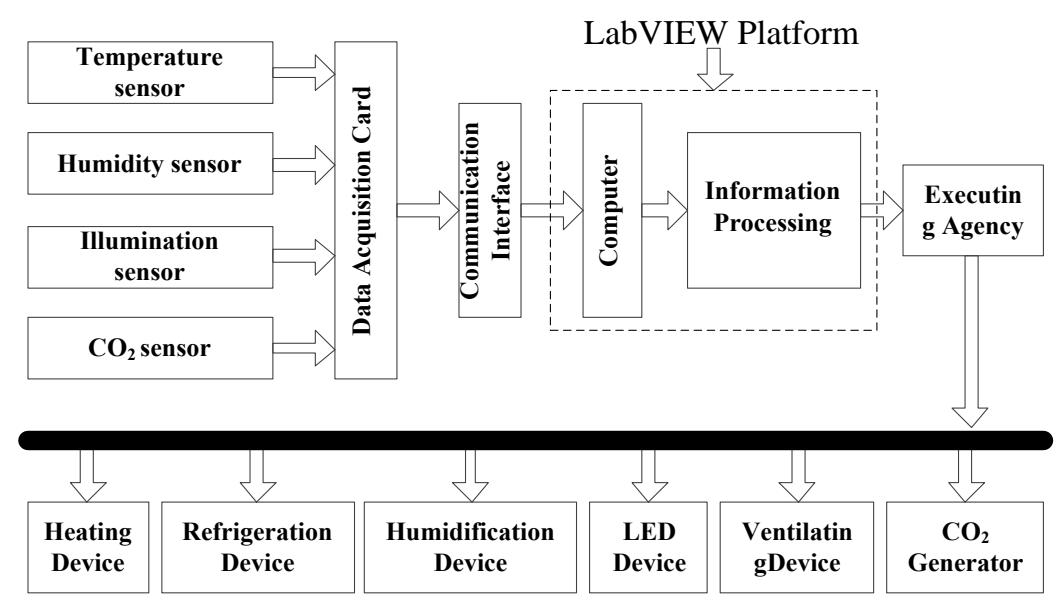

Fig.1: Structural diagram of control system

The overall scheme design of the plant growth environment control system mainly includes two parts: the software and hardware. The hardware module is mainly composed of three parts: the plant growth cabinet module, the sensor module and the actuator module; the software module is mainly composed of the data acquisition module, data analysis module and data prediction module.

\subsection{Design of hardware module}

\subsubsection{Sensor module}

1.Temperature and humidity sensor: The temperature and humidity sensor use a sensor with a SHT11 chip, which internally contains a 14-bit A/D converter and uses a quasi-I2C interface to transmit data. Here, software is used to simulate $\mathrm{I} 2 \mathrm{C}$ communication sequence to receive data, and the function of calibrating digital signal is also included in the sensor.

2. Illumination sensor: The light intensity sensor HSTL-GZD-20W uses a high-sensitivity silicon-blue photovoltaic detector as the photoreceptor, and the output is $0 \sim 5 \mathrm{~V}$.

\section{3. $\mathrm{CO}_{2}$ concentration sensor}

The $\mathrm{CO}_{2}$ concentration sensor selects low power infrared carbon dioxide sensor COZIR-ambient. The sensor has the function of temperature, humidity and light. The maximum measurement range is from 2000 ppm to $10000 \mathrm{ppm}$, and the accuracy can reach $1 \%$.

\subsubsection{Actuator module}

The execution module in the plant growth environment control system is mainly composed of environmental adjustment devices such as heating rods, ventilators, environmental chillers, humidifiers, etc., and is used to regulate various environmental factors of plant growth, and the control mode is realized by a relay.

\subsubsection{NI data acquisition card}

In the plant growth environment control system, the collected environmental factors are all analog quantities, and the computer can only analyze and calculate the digital quantities. So the data acquisition card is used to convert the collected analog quantity into digital quantity for computer analysis and processing. This article uses a DAQ board, model number NI PCIe-6351. 


\subsection{Design of software module}

Based on the LabVIEW software platform, the software module is designed to control the plant growth environment control system. It can display the plant growth environment factor parameters detected by the sensor in real time, and implement the precise control of environmental factors according to the BP neural network plant growth environment state model.

\section{Analysis and Experimental Study on the Influence of Environmental Factors on Plant Growth}

In the natural environment, plant growth environment is unstable, and weather conditions such as seasonal change, wind and rain lead to slow growth, long cycle and low yield of plants. In order to study the growth status of plants under different environmental conditions, a controllable plant growth environment is established in this paper: from four aspects of temperature, humidity, illumination and $\mathrm{CO}_{2}$ concentration to study the changes of environment on plant growth. The changes of environmental factors under different conditions were measured, and compared with the set environmental factors, the promotion effect of controlled environment on plant growth was obtained. The measurement results of four environmental factors under different conditions are shown in figure2 (a), (b), (c) and (d), respectively.

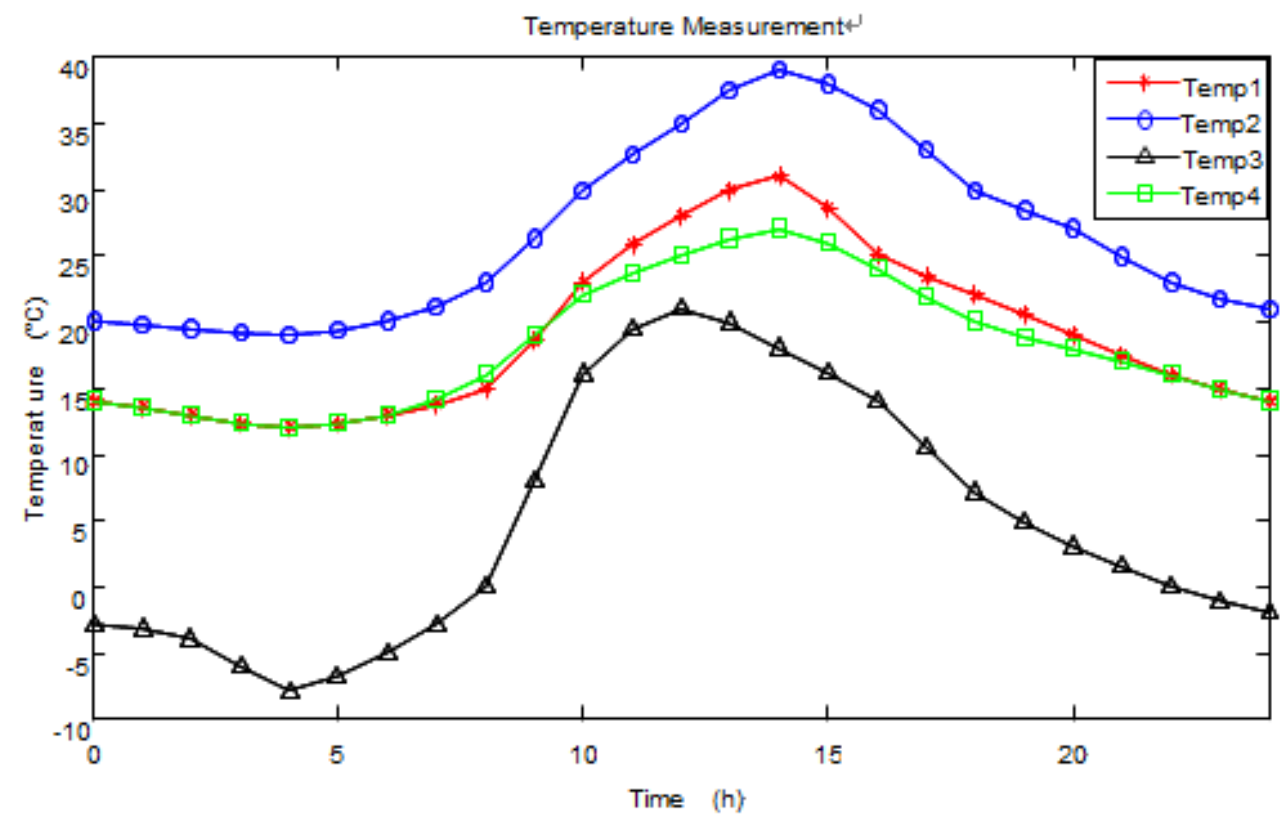

(a)

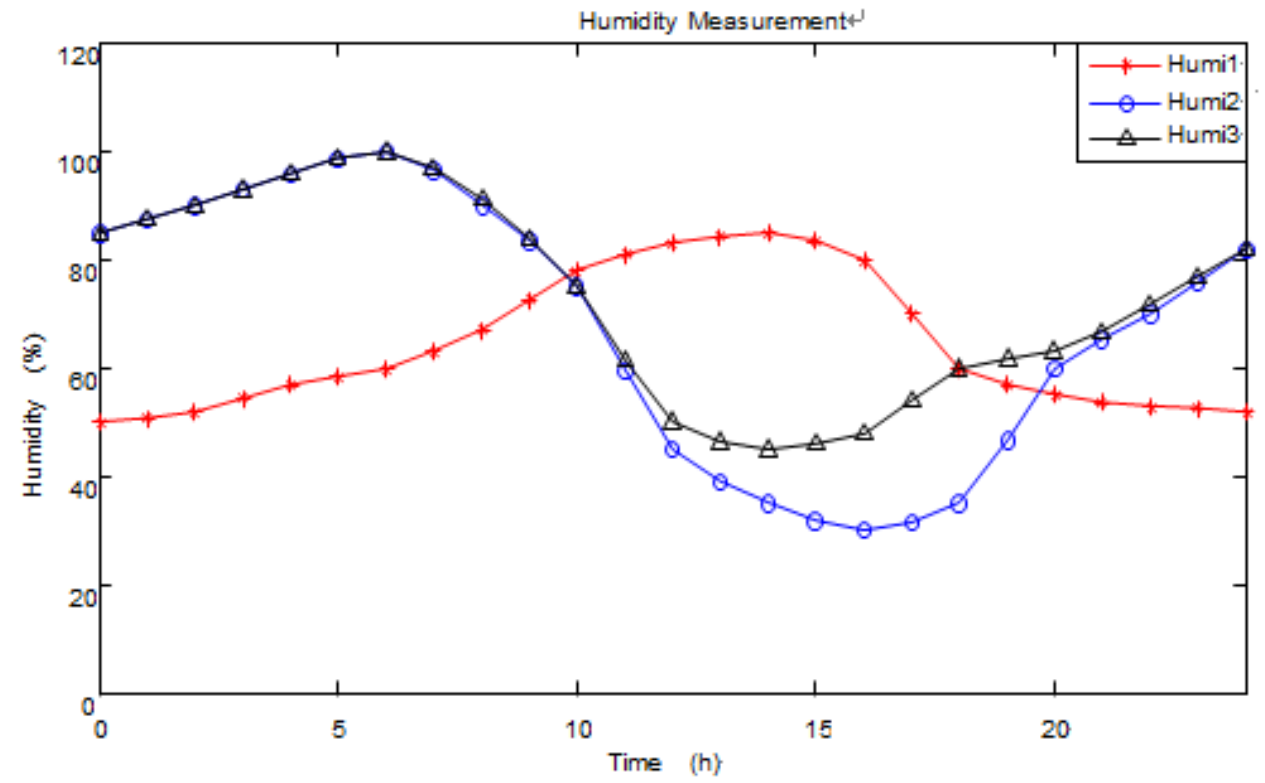

(b) 


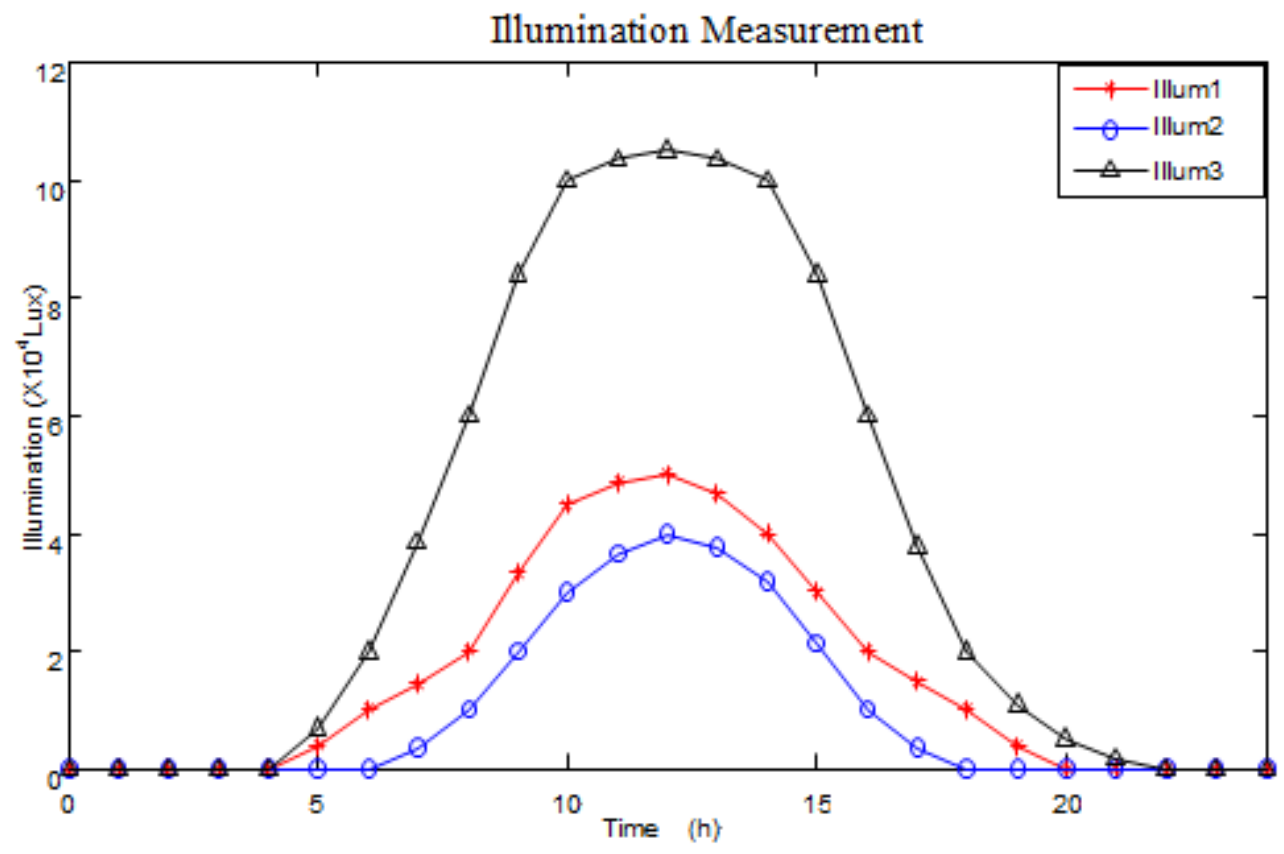

(c)

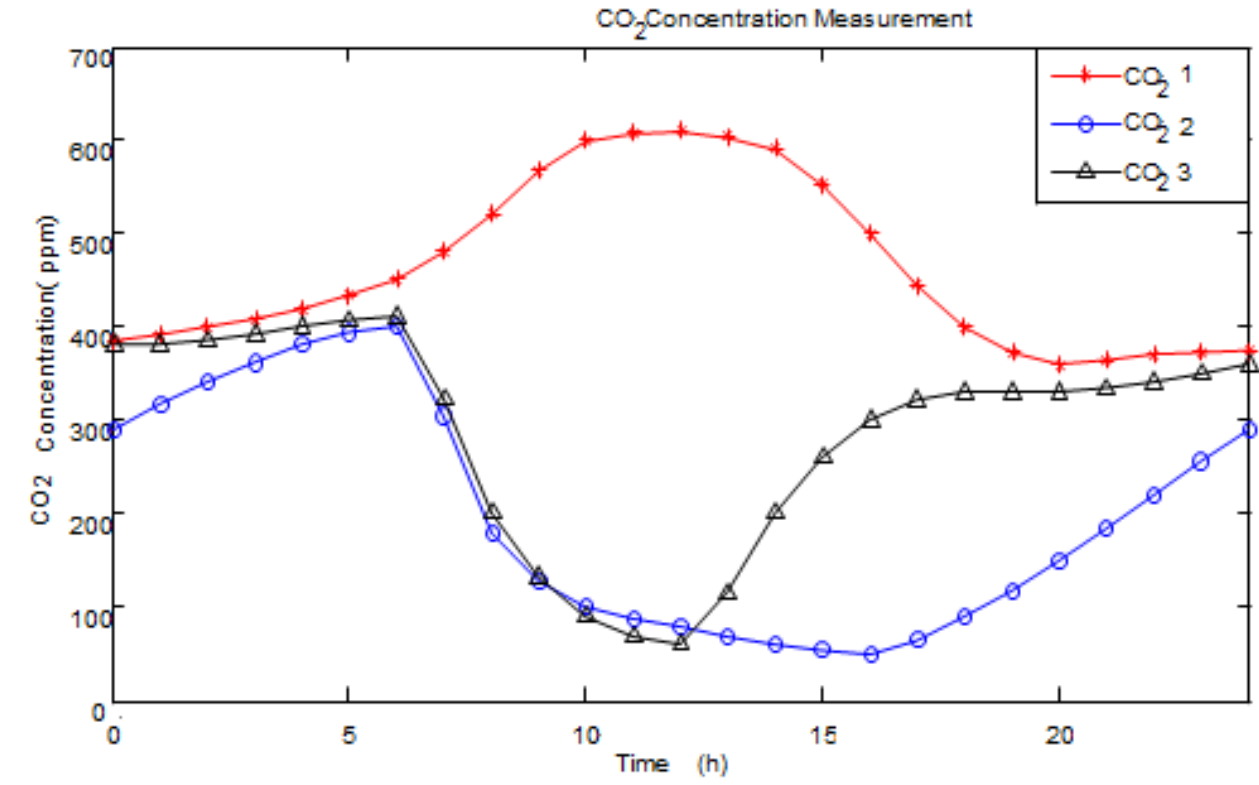

(d)

Fig.2: Measurement results of four environmental factors under different conditions

In the experiment of periodic variation of temperature, the temperature is controlled comprehensively by adjusting heating rod, ventilation fan and other ways. The temperature changes under four different conditions are analyzed, which are controlled environment system, high temperature in summer, cold in winter and adjusting ventilation fan only.

In the experiment of the relative humidity cycle, the humidity of the growth cabinet was adjusted by two methods, such as spray humidifier, ventilator, and so on. The humidity in the growth cabinet was adjusted by controlling the humidity of the ventilator only. The change of humidity under different conditions in 3 was analyzed.

In the comprehensive analysis of illumination, the illumination is regulated by LED light plate, and the change rule of illumination intensity under three different conditions is analyzed, which are: the change of illumination in controllable environment system, the short illumination time in winter and the long illumination time in summer. 
In the experiment of periodic variation of $\mathrm{CO}_{2}$ concentration, $\mathrm{CO}_{2}$ generator and ventilator were used to control the change of $\mathrm{CO}_{2}$ concentration comprehensively. The changes of $\mathrm{CO}_{2}$ concentration under three different conditions were analyzed, namely, in the controllable environment system, natural conditions, supplementing $\mathrm{CO}_{2}$ only by ventilator, and the changes of $\mathrm{CO}_{2}$ concentration in plant growth cabinet.

\section{Plant Growth Environment Control Model Based on BP Neural Network}

\subsection{BP neural network}

BP neural network, also known as multi-layer perceptron, can self-adjust parameters and establish the corresponding relationship between input and output in the absence of a mathematical model.

Because BP network adopts supervised learning, it is necessary to design its structure when solving a specific problem by using BP network, including network layer, number of input neurons, number of hidden neurons, number of output neurons and transfer function.

The application process of BP neural network can be divided into two steps: the first step is to calculate the input signal through the input layer and the hidden layer, and the result reaches the output layer to get the output value; the second step is to reverse-propagate the error. When the output value can not meet the expected value, the weight coefficients are adjusted step by step until the output value minus the expected value meets the error requirements.

The input and output of the network input layer are:

$$
o_{j}{ }^{(1)}(k)=x(j)(j=1,2, \ldots, n)
$$

The number $\mathrm{n}$ of input variables in the formula depends on the complexity of the controlled system.

The input and output of the network hidden layer are:

$$
\left\{\begin{array}{l}
n e t_{i}^{(2)}(k)=\sum_{j=1}^{m} w_{j i}{ }^{(2)} o_{j}{ }^{(1)} \\
o_{i}^{(2)}(k)=f\left(n e t_{i}^{(2)}(k)\right)(i=1,2, \cdots, q)
\end{array}\right.
$$

The input and output of the network output layer are:

$$
\left\{\begin{array}{l}
\text { net }_{i}{ }^{(3)}=\sum_{i=0}^{q} W_{j i}{ }^{(3)} o_{j}{ }^{(2)}(k) \\
o_{1}{ }^{(3)}(k)=g\left(\text { net }_{i}{ }^{(3)}(k)\right),(1=1,2,3,4) \\
O_{1}{ }^{(3)}(k)=Y_{1} \\
o_{2}{ }^{(3)}(k)=Y_{2} \\
o_{3}{ }^{(3)}(k)=Y_{3} \\
o_{4}{ }^{(3)}(k)=Y_{4}
\end{array}\right.
$$

The output layer node is the plant growth environment state.

Where is the weighting coefficient of the hidden layer; the upper corners (1), (2), and (3) represent the input layer, the hidden layer, and the output layer, respectively.

The activation function of the hidden layer neurons takes a positive and negative symmetric function:

$$
f(x)=\tanh (x)=\frac{e^{x}-e^{-x}}{e^{x}+e^{-x}}
$$

Since all are positive numbers, the neuron activation function of the output layer takes a non-negative function, ensuring that the output value must be positive:

$$
g(x)=\frac{1}{2}(1+\tanh (x))=\frac{e^{x}}{e^{x}+e^{-x}}
$$


Take the performance indicator function as:

$$
\mathrm{e}(k)=\frac{1}{2}(r(t)-c(t))^{2}
$$

\subsection{State model of plant growth environment}

Plant growth depends on the external environment, which needs to provide the necessary environmental conditions such as temperature, humidity, illumination and $\mathrm{CO}_{2}$ concentration for its growth. To realize the regulation and control of plant growth environment, it is necessary to determine the state of plant growth environment.

According to the relationship between environmental factors and plant growth obtained in the previous article, the plant growth environment state is divided into 9 types: normal temperature, high humidity, low temperature, high humidity, high temperature, high humidity, normal temperature, normal humidity, low temperature, low temperature, temperature Normal humidity is high, temperature is low, humidity is normal, temperature is low, humidity is low, temperature is low, and humidity is high. To this end, we build a neural network structure as shown in figure3.

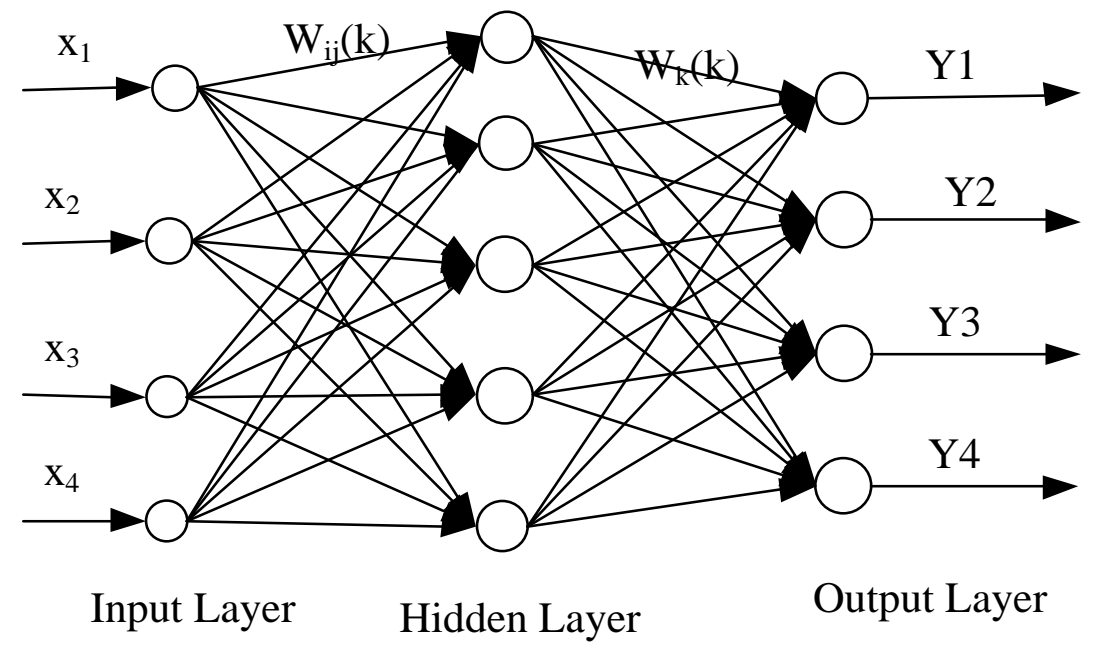

Fig.3: Structural chart of neural network

The input nodes of the BP neural network are selected as needed, namely temperature $\mathrm{x} 1$, humidity $\mathrm{x}$, illuminance $\mathrm{x} 3$, and $\mathrm{CO}_{2}$ concentration $\mathrm{x} 4$. The target output modes of the nine growth environment states are (0000), (0001), (0010), (0011), (0100), (0101), (0110), (0111), (1000), one-to-one correspondence with the nine states of the output, therefore, the number of neurons of the output layer is set to 4, Y1, Y2, Y3, Y4 respectively take a value of 0 or 1 , representing the state of the environmental factor.

\section{Implementation of plant growth environment control system}

Intelligent plant growth system, which is a complex control system with multi-parameter control. In this paper, the adjustment method of parameter order judgment is adopted. Firstly, the ambient temperature and humidity are adjusted, and then the illuminance is adjusted. According to the set value and the sensor detection value, the judgment is made in order, and the corresponding regulation is made, the purpose is to reduce the energy consumption and reduce the complexity of the system control.

Figure4 shows the control scheme of the plant growth environment control system. 


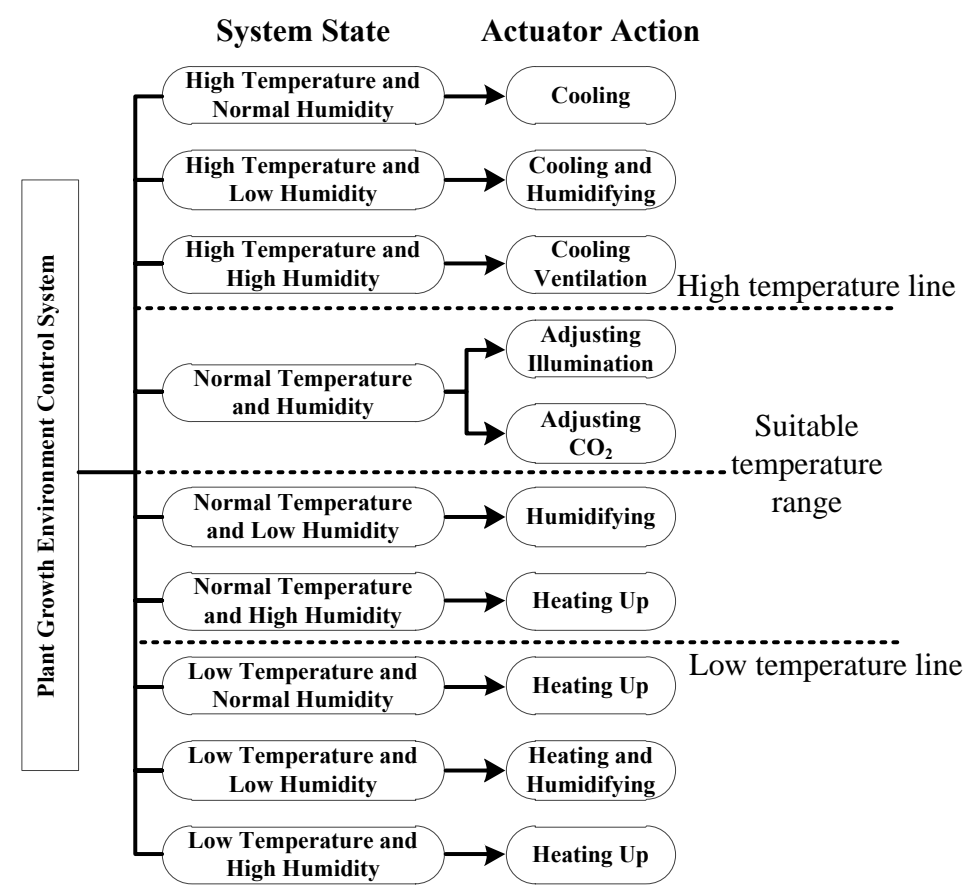

Fig.4: Control scheme chart of plant growth environment control system

According to the constructed plant growth environment state model, the environmental state is judged, the actuator is adjusted, and the plant growth environment is adjusted. Because the laboratory equipment is a switch-controlled actuator, in order to extend the life of the actuator, it is not possible to switch it frequently, so it is necessary to change the adjustment method.

The method adopted in this paper is step-by-step adjustment. The temperature range in the plant growth cabinet is $2{ }^{\circ} \mathrm{C}$, the humidity variation range is $60 \%$, and the illumination variation range is $400 \mathrm{ppm}$. In this experiment, the standard temperature was set to $20^{\circ} \mathrm{C}$, the relative humidity standard value was $50 \%$, and the illuminance standard value was $1400 \mathrm{ppm}$. The range of changes in the set value: temperature: $19 \sim 21^{\circ}$ C, humidity: 20\% 80\%, illuminance: 1200ppm 1600ppm.

\section{Conclusion}

Intelligent agriculture has raised much attention, and the problem of waste of energy has come one after another. How to save energy is a problem that needs to be solved under the premise of realizing the function of intelligent agriculture. Based on the full investigation of the optimal control of plant growth environment at home and abroad, this paper proposes and establishes a plant growth environment control model based on BP neural network, and uses the micro-plant growth system as the research basis, using the method of first simulation and post-validation. Go to the control system of the plant growth system. The experimental results show that BP neural network technology has a good application value in plant growth environment control, and has important significance for the research and development of intelligent agriculture.

\section{Acknowlegements}

This work was supported by the Porgram for Discipline Leading Talents.

\section{References}

[1] Zhiqi Liu, Liguo Tian, Meng Li, Jianglin Wei, Gaoli Chen, Yushuang LiStudy for PID Temperature Control of Plant Growth Cabinet Based on Neural Network[C]. Electronics, Automation and Engineering of Power Systems, 2015.

[2] CHU L, KANG Y, WAN S. Effects of water application intensity of microsprinkler irrigation on water and salt environment and crop growth in coastal saline soils[J]. Journal of Integrative Agriculture, 2015, 14(10): 20772089. 
[3] Seginer I. Some artificial neural network applications to greenhouse environmental control[J]. Computers and Electronics in Agriculture, 1997, 18(2): 167-186.

[4] Daniel J, Andrés P U, Héctor S, et al. A survey of artificial neural network-based modeling in agroecology[M]. Soft Computing applications in industry. Springer Berlin Heidelberg, 2008: 247-269.

[5] Aaslyng J M, Ehler N, Jakobsen L. Climate control software integration with a greenhouse environmental control computer[J]. Environmental Modelling \& Software, 2005, 20(5): 521-527.

[6] Qu Y, Ning D, Lai Z, et al. Neural networks based on PID control for greenhouse temperature[J]. Transactions of the Chinese Society of Agricultural Engineering, 2011, 27(2): 307-311.

[7] Yu H, Zhang G, Lu N. Information Collection System of Crop Growth Environment Based on the Internet of Things[J]. Asian Agricultural Research, 2013, 5(05):148-150.

[8] Baek M S, Kwon S Y, Lim J H. Improvement of uniformity in cultivation environment and crop growth rate by hybrid control of air flow devices[J]. Journal of Central South University, 2015, 22(12): 4702-4708.

[9] YE Maozhi. Application of BP Neural Network in Tremella Cultivation Environmental Control[J]. Journal of Xinzhou Teachers University,2015,31(2):17-19.

[10] Wang Xi, Wang Xiaozhi, Lin Weiguo. Research on improved regulator model of greenhouse light environment based on neural network[J].Journal of Chinese Agricultural Mechanization,2016,37(6):69-74. 